\title{
Rg-HX 分子间势的精确从头计算研究
}

\author{
张 愚史鸿运王伟周 \\ (贵州大学化学系, 贵阳 550025)
}

\begin{abstract}
摘要 在用非迭代的三重激发项来校正 CCSD 的 CCSD ( T) 理论水平下,采用 aug-cc- $p V Q Z$ 基函数对 $\mathrm{He}-\mathrm{HF}$ 的分 子间势进行了系统的研究. 结果表明: He-HF 以线型结构存在. 在极限基的情况下, 复合物两种线型极小点结构 He-H-F 和 He-F-H 势阱深分别为 $46.614 \mathrm{~cm}^{-1}$ 和 $25.026 \mathrm{~cm}^{-1}$, 对应 $\mathrm{He}$ 原子到 $\mathrm{HF}$ 分子质心的距离 $R_{\mathrm{m}}$ 分别为 $0.3149 \mathrm{~nm}$ 和 $0.3012 \mathrm{~nm}$. 讨论了不同的基函数和理论方法在研究此类弱束缚态复合物的分子间势时的可靠性 及其对结果的影响, 并研究了 $\mathrm{HF}$ 分子中 $\mathrm{H}-\mathrm{F}$ 键长的改变对势能的影响, 同时也给出了势函数的解析形式.
\end{abstract}

关键词：从头计算, 分子间势, $\mathrm{PES}, \mathrm{CBS}$

稀有气体 - 氢卢酸复合物与氢键相比, 它们之 间的相互作用力要弱得多, 因而, 其研究要求精度 更高. 长期以来，为了探索分子间相互作用力的本 质及分子间或分子内动力学的详细情况，几何结构 简单的三原子稀有气体 - 氢卤酸复合物一直是人们 研究的中心和热点之一。人们对 Xe、 Kr、Ar 以及 $\mathrm{He} 、 \mathrm{Ne}$ 与 $\mathrm{HF} 、 \mathrm{HCl} 、 \mathrm{HBr}$ 的复合物进行的大量系统 的量子化学从头计算和实验方面的研究 ${ }^{[1-2]}$, 已经 给出了有关它们结构和内部动力学的丰富信息. 这对分子间相互作用力的研究和发展起了重要的 推动作用. 以前, 确定弱的相互作用分子体系的分 子间势主要是从实验 (如原子 - 分子碰撞、光谱、输 运过程和气体状态方程等) 获得。近年来, 随着量 子化学理论和计算机技术的发展, 第一性原理计算 已成为获取分子间势的另一重要途径. 但是, 由于 对分子间势进行量子化学从头计算, 不仅要求采用 大的极化、弥散基组, 而且还要在考虑电子相关能 的理论级别下进行. 所以目前通过精确量子化学 从头算来确定分子间相互作用势只限于一些较小 的体系. $\operatorname{Rg}(\mathrm{Rg}=\mathrm{He} 、 \mathrm{Ne} 、 \mathrm{Ar} 、 \mathrm{Ke} 、 \mathrm{Xe})-\mathrm{HX}(\mathrm{X}=\mathrm{F}$ 、 $\mathrm{Cl} 、 \mathrm{Br})$ 正是这样的几个 标准”的体系.

和其它的稀有气体 $(\mathrm{Ar} 、 \mathrm{Ke} 、 \mathrm{Xe})$ 相比, He、 $\mathrm{Ne}$ 与 $\mathrm{HF} 、 \mathrm{HCl} 、 \mathrm{HBr}$ 之间的相互作用要更弱一些. 这
是因为 $\mathrm{He}$ 的极化能力仅为 $\mathrm{Ar}$ 的八分之一, $\mathrm{Ne}$ 的 极化能力为 $\mathrm{Ar}$ 的四分之一 ${ }^{[3]}$, 比 $\mathrm{Ke} 、 \mathrm{Xe}$ 的极化能 力更小, 因此产生分子间相互作用势吸引部分的诱 导和弥散力更小一些, 分子间势相应更为狭窄. 由 于结合能非常小, 研究重稀有气体复合物时的实验 技术很难成功地应用到 $\mathrm{He} 、 \mathrm{Ne}$ 与 $\mathrm{HF} 、 \mathrm{HCl} 、 \mathrm{HBr}$ 形 成的复合物上. 因此, 与其它稀有气体相比, He、 $\mathrm{Ne}$ 复合物的实验研究在时间上要推后一点, 数量上

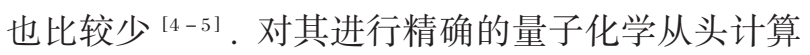
就显得尤为重要. Hutson 研究组 ${ }^{[1,6-10]}$ 在这方面作 了大量的、系统的工作, 他们主要是通过构筑经验 势拟合实验数据来进行研究. 我们通过对美国 Wesleyan 大学弱束缚分子复合物数据库及其它大 量文献的详细查询得知 ${ }^{[11]}$, 对于 $\mathrm{Ar} 、 \mathrm{Ke} 、 \mathrm{Xe}$ 与卤化 氢形成的复合物无论是实验方面或者是量子化学 从头计算理论方面都有大量的研究文献报导, 而对 于 $\mathrm{He}, \mathrm{Ne}$ 与卤化氢形成的复合物研究则比较少 见, 未见有高级的量子化学从头计算研究的报导. 为了得到 He-HF、He-HCl、Ne-HF、 $\mathrm{Ne}-\mathrm{HCl} 、 \mathrm{He}-\mathrm{HBr}$ 、 $\mathrm{Ne}-\mathrm{HBr}$ 体系精确的分子间势, 从而对其进行研究, 以探讨其本质和规律性，作者用非迭代的三重激发 项来校正 CCSD 的 CCSD ( T) 理论方法 ${ }^{[20]}$ 和大基组 研究了该体系的分子间相互作用势并拟合成解析

2001-06-18 收到初稿，2001-08-10 收到修改稿. 联系人:史鸿运 (E-mail: hyshi@ tmail. gzu. edu. cn). **贵州省教委及科委基金资助 项目 
势能函数.

此项研究有以下两个目的：(1) 给出 He-HF、 $\mathrm{He}-\mathrm{HCl} 、 \mathrm{Ne}-\mathrm{HF} 、 \mathrm{Ne}-\mathrm{HCl} 、 \mathrm{He}-\mathrm{HBr} 、 \mathrm{Ne}-\mathrm{HBr}$ 复合物基 态时高质量的势能面, (2)讨论不同的基函数和理论 方法在研究此类弱束缚态复合物的分子间势时的可 靠性及其对结果的影响, 同时也给出了势函数的解 析形式, 并讨论了 $\mathrm{HF}$ 分子中 $\mathrm{H}-\mathrm{F}$ 键长的改变、 $\mathrm{HCl}$ 分子中 $\mathrm{H}-\mathrm{Cl}$ 键长的改变、 $\mathrm{HBr}$ 分子中 $\mathrm{H}-\mathrm{Br}$ 键长的改变对势能的影响. 最后也对各个复合物的 分子间势的特点进行了归纳和对比. 本文仅发表对 $\mathrm{He}-\mathrm{HF}$ 分子间势进行精确的量子化学从头计算研究 的结果.

\section{1 计算方法}

如前所述, 稀有气体原子 $\mathrm{He}$ 与 $\mathrm{HF}$ 分子之间 的相互作用属于非常弱的分子间相互作用范畴。对 于它们的研究, 必须采用非常精确的量子化学计算 方法. 本文的研究采用了超分子方法. 也就是说, 某一给定理论级别下的分子间相互作用能 $\Delta E$ 通过 下式计算获得：

$$
\Delta E=E_{\mathrm{AB}}-E_{\mathrm{A}}-E_{\mathrm{B}}
$$

式中 $E_{\mathrm{AB}}$ 是复合物的能量, $E_{\mathrm{A}}$ 和 $E_{\mathrm{B}}$ 分别是单体 A、B 的能量. 众所周知, 对于稀有气体复合物来 讲, Hartree-Fock 相互作用势是排斥性质的, 而弥散 能在稀有气体复合物分子间相互作用势能中占主要 的部分. 因此, 在对 He-HF 分子间势进行计算时, 精确的相关处理是非常重要的. 本文给出的计算结 果中, 上标表示所用的理论级别, 即 $\Delta E^{\mathrm{CCSD}(\mathrm{T})}$ 表示 $\operatorname{CCSD}(\mathrm{T})$ 相互作用能. 最精确的结果是 $\operatorname{CCSD}(\mathrm{T})$ 值 ${ }^{[12]}$. 同时, 我们也给出了在 MP2 和 CCSD 理论级 别下的相互作用能数值, 以考察理论方法的改变对 此类分子间弱相互作用能值的影响. 基函数选取 Dunning 等人的增大了极化函数的相关连续性四 $\zeta$ 基组（aug-cc- $p V Q Z$ ）. 当我们考察基函数的变化对 相互作用能值的影响时, 选取基组一般形式为 aug-cc- $p V n Z$, 其中 $n=D, T, Q, 5$ 等, 相应为双 $\zeta$ 基、三 $\zeta$ 基、四 $\zeta$ 基等 ${ }^{[13-15]}$. 同时也计算了完全基
(CBS $)^{[16-17]}$. 由于在量子化学计算中所采用的基组 是一个有限基组, 所以在计算分子间相互作用能时, 计算单体时所使用的基组与计算整个体系时总的基 函数相比是一个比较小的数目, 这种基组上的不一 致性导致了所谓的基组重叠误差（BSSE）。它常常 对分子间相互作用能的计算结果带来较大的影响. 为了消除 BSSE, 我们采用 Boys 和 Berbnadi 提出的 $\mathrm{CP}$ 方法 (Counterpoise method) ${ }^{[18]}$.

在本文的 He-HF van der Waals 复合物的计算 中, 采用标准的 Jacobi 坐标系 $(\boldsymbol{R}, \boldsymbol{r}, \theta)$, 矢量 $\boldsymbol{r}$ 方 向是从 $\mathrm{F}$ 原子核中心位置到 $\mathrm{H}$ 原子核中心位置, 长 度值为 $r$, 矢量 $\boldsymbol{R}_{\mathrm{m}}$ 方向是从 $\mathrm{HF}$ 质心位置到 $\mathrm{He}$ 原 子核中心位置, 长度值为 $R_{\mathrm{m}}$. 矢量 $\boldsymbol{R}_{\mathrm{m}}$ 和矢量 $\boldsymbol{r}$ 之 间的夹角定义为 $\theta, \theta=0^{\circ}$ 时对应线型构型 $\mathrm{He}-$ $\mathrm{HF}$. 由于实验上 $\mathrm{HF}$ 分子中两原子间平衡距离还没 有确定, 在计算过程中, $r$ 值都固定, 采用 CCSD (T) / aug-cc- $p V Q Z$ 下的优化值 $r_{\mathrm{HF}}=0.0918 \mathrm{~nm}$.

本文全部计算采用 GAUSSIAN 94 量子化学计 算软件包 ${ }^{[19]}$, 在贵州大学高性能计算化学实验室 (GHPCC) 美国 Intel 公司的 Pentium II/450 UNIX 高速工作站上完成。

\section{2 计算结果与讨论}

\subsection{He-HF 的二维势能面}

在 $\operatorname{CCSD}(\mathrm{T}) /$ aug-cc- $p V Q Z$ 理论水平下, 不同 距离、角度的复合物 He-HF 的相互作用能列于表 1 . 本文给出了详细的结果, 以便其他的研究人员用这 些原始的数据去建立自己的模型势. 计算过程中, 分子间相互作用距离 $R_{\mathrm{m}}$ 取值范围为 $0.250 \sim 0.380$ $\mathrm{nm}$, 角度 $\theta$ 取 $0^{\circ} \sim 180^{\circ}$ 范围内间隔为 $15^{\circ}$ 的 11 个数 值. 通过对 $\theta=0^{\circ} 、 90^{\circ} 、 180^{\circ} 3$ 个角度的相互作用能 作 3 次样条函数插值, 得到各个角度下 $V_{\mathrm{m}}$ 的极小 值 $V_{\min }$ 和对应的 $R_{\mathrm{m}}$ 值的 $R_{\min }$ (见表 2). 图 1 描绘了 该势能面的等势图.

从表 2 和图 1 我们可以看到, 这个势能面最明 显的特征是其各向异性不是非常明显. 在 CCSD (T) 势能面上存在两个极小值, 都为线型结构, 第一 
表 $1 \mathrm{He}-\mathrm{HF}$ 复合物在不同距离、理论水平下的相互作用能

Table 1 Interaction energies of the He-HF complex at different distances and theory levels

\begin{tabular}{|c|c|c|c|c|c|}
\hline$\theta /\left({ }^{\circ}\right)$ & $R_{\mathrm{m}} / \mathrm{nm}$ & $\Delta E^{\mathrm{HF}} / \mathrm{cm}^{-1}$ & $\Delta E^{\mathrm{MP} 2} / \mathrm{cm}^{-1}$ & $\Delta E^{\mathrm{CCSD}} / \mathrm{cm}^{-1}$ & $\Delta E^{\mathrm{CCSD}(\mathrm{T})} / \mathrm{cm}^{-1}$ \\
\hline \multirow[t]{9}{*}{0} & 0.250 & 527.448 & 348.864 & 338. 353 & 314.624 \\
\hline & 0.270 & 203.758 & 89. 386 & 79. 112 & 63. 199 \\
\hline & 0.290 & 71. 266 & -3.169 & -11.171 & -21.876 \\
\hline & 0.310 & 19. 685 & -29.402 & -35.075 & -42.296 \\
\hline & 0.316 & 11.937 & -31.491 & -36.558 & -42.979 \\
\hline & 0.320 & 7. 989 & -32.058 & -36.750 & -42.689 \\
\hline & 0.340 & -2.425 & -29.338 & -32.494 & -36.528 \\
\hline & 0.360 & -4.970 & -23.318 & -25.427 & -28.192 \\
\hline & 0.380 & -4.862 & -15.572 & -18.990 & -20.907 \\
\hline \multirow[t]{9}{*}{20} & 0.250 & 463.701 & 304.154 & 290.780 & 269.734 \\
\hline & 0.270 & 182.297 & 79. 519 & 68.330 & 54. 144 \\
\hline & 0.290 & 65.811 & -1.522 & -9.712 & -19.320 \\
\hline & 0.310 & 19. 720 & -24.989 & -30.620 & -37.149 \\
\hline & 0.316 & 12.673 & -26.965 & -31.965 & -37.783 \\
\hline & 0.320 & 9.050 & -27.553 & -32.167 & -37.556 \\
\hline & 0.340 & -0.773 & -25.546 & -28.614 & -32.304 \\
\hline & 0.360 & -3.506 & -20.514 & -22.552 & -25.100 \\
\hline & 0.380 & -3.742 & -15.602 & -16.969 & -18.750 \\
\hline \multirow[t]{9}{*}{40} & 0.250 & 347.550 & 228.492 & 209.820 & 194.418 \\
\hline & 0.270 & 142.373 & 64.085 & 51. 108 & 40.491 \\
\hline & 0.290 & 55.353 & 2.950 & -5.674 & -13.036 \\
\hline & 0.310 & 19.545 & -16.007 & -21.618 & -26.738 \\
\hline & 0.316 & 13.825 & -17.896 & -22.819 & -27.414 \\
\hline & 0.320 & 10.822 & -18.594 & -23.106 & -27.382 \\
\hline & 0.340 & 2. 107 & -18.151 & -21.076 & -24.068 \\
\hline & 0.360 & -0.828 & -15.046 & -16.961 & -19.071 \\
\hline & 0.380 & -1.634 & -11.717 & -12.993 & -14.493 \\
\hline \multirow[t]{9}{*}{60} & 0.250 & 275.020 & 189. 558 & 167.259 & 156. 309 \\
\hline & 0.270 & 117.316 & 59. 228 & 44. 948 & 37. 116 \\
\hline & 0.290 & 48.814 & 8. 666 & -0.324 & -5.934 \\
\hline & 0.310 & 19. 521 & -8.529 & -14.163 & -18.179 \\
\hline & 0.316 & 14. 652 & -10.582 & -15.482 & -19.114 \\
\hline & 0.320 & 12.049 & -11.475 & -15.942 & -19.339 \\
\hline & 0.340 & 4. 182 & -12.269 & -15.302 & -17.736 \\
\hline & 0.360 & 1.046 & -10.848 & -12.676 & -14.425 \\
\hline & 0.380 & -0.119 & -8.701 & -9.907 & -11.173 \\
\hline \multirow[t]{9}{*}{80} & 0.250 & 254. 138 & 183.347 & 160.492 & 151.317 \\
\hline & 0.270 & 110.237 & 61.112 & 46. 781 & 40.110 \\
\hline & 0.290 & 47. 108 & 12.512 & 3.618 & -1.227 \\
\hline & 0.310 & 19. 701 & -4.878 & -10.387 & -13.896 \\
\hline & 0.316 & 15.078 & -7.132 & -11.909 & -15.093 \\
\hline & 0.320 & 12.591 & -8.173 & -12.520 & -15.504 \\
\hline & 0.340 & 4. 949 & -9.933 & -12.667 & -14.823 \\
\hline & 0.360 & 1.764 & -8.971 & -10.723 & -12.284 \\
\hline & 0.380 & 0.479 & -7.327 & -8.447 & -9.613 \\
\hline
\end{tabular}


Cont. table 1

\begin{tabular}{|c|c|c|c|c|c|}
\hline$\theta /\left({ }^{\circ}\right)$ & $R_{\mathrm{m}} / \mathrm{nm}$ & $\Delta E^{\mathrm{HF}} / \mathrm{cm}^{-1}$ & $\Delta E^{\mathrm{MP} 2} / \mathrm{cm}^{-1}$ & $\Delta E^{\mathrm{CCSD}} / \mathrm{cm}^{-1}$ & $\Delta E^{\mathrm{CCSD}(\mathrm{T})} / \mathrm{cm}^{-1}$ \\
\hline \multirow[t]{9}{*}{90} & 0.250 & 252.607 & 182.89 & 160.887 & 151.833 \\
\hline & 0.270 & 109.506 & 61.221 & 47. 398 & 40.852 \\
\hline & 0.290 & 46. 801 & 12. 833 & 4. 251 & -0.483 \\
\hline & 0.310 & 19.600 & -4.513 & -9.834 & -13.253 \\
\hline & 0.316 & 15. 013 & -6.771 & -11.387 & -14.486 \\
\hline & 0.320 & 12.546 & -7.818 & -12.018 & -14.922 \\
\hline & 0. 340 & 4.965 & -9.624 & -12.266 & -14.360 \\
\hline & 0.360 & 1. 805 & -8.715 & -10.408 & -11.923 \\
\hline & 0.380 & 0.526 & -7.123 & -8.234 & -9.336 \\
\hline \multirow[t]{9}{*}{100} & 0.250 & 251.627 & 180.037 & 159.528 & 150.289 \\
\hline & 0.270 & 108. 593 & 59.451 & 46. 493 & 39.891 \\
\hline & 0.290 & 46. 172 & 11.865 & 3. 783 & -0.945 \\
\hline & 0.310 & 19. 212 & -4.993 & -10.023 & -13.412 \\
\hline & 0.316 & 14.681 & -7.153 & -11.520 & -14.587 \\
\hline & 0.320 & 12.247 & -8.144 & -12.121 & -14.990 \\
\hline & 0.340 & 4. 793 & -9.758 & -12.266 & -14.326 \\
\hline & 0.360 & 1.709 & -8.756 & -10.367 & -11.853 \\
\hline & 0.380 & 0.474 & -7.123 & -8.182 & -9.262 \\
\hline \multirow[t]{9}{*}{120} & 0.250 & 242.245 & 161.661 & 145.512 & 135. 470 \\
\hline & 0.270 & 102.883 & 49. 083 & 38.664 & 31.705 \\
\hline & 0.290 & 42.902 & 6.267 & -0.342 & -5.199 \\
\hline & 0.310 & 17. 388 & -7.942 & -12.112 & -15.523 \\
\hline & 0.316 & 13. 152 & -9.579 & -13.213 & -16.286 \\
\hline & 0.320 & 10. 888 & -10.274 & -13.591 & -16.457 \\
\hline & 0.340 & 4. 034 & -10.870 & -12.986 & -15.199 \\
\hline & 0.360 & 1. 276 & -9.346 & -10.719 & -12.174 \\
\hline & 0.380 & 0.216 & -7.447 & -8.358 & -9.410 \\
\hline \multirow[t]{9}{*}{140} & 0.250 & 220.384 & 128.614 & 117.414 & 106. 496 \\
\hline & 0.270 & 91.569 & 31.866 & 24.400 & 17. 032 \\
\hline & 0. 290 & 37. 174 & -2.492 & -7.349 & -12.373 \\
\hline & 0.310 & 14.517 & -12.335 & -15.465 & -18.926 \\
\hline & 0.316 & 10.819 & -13.148 & -15.891 & -18.993 \\
\hline & 0.320 & 8. 855 & -13.381 & -15.894 & -18.779 \\
\hline & 0.340 & 3.003 & -12.432 & -14.064 & -16.085 \\
\hline & 0.360 & 0.736 & -10.15 & -11.225 & -12.660 \\
\hline & 0.380 & -0.081 & -7.879 & -8.603 & -9.635 \\
\hline \multirow[t]{9}{*}{160} & 0.250 & 196.429 & 95.836 & 88.536 & 76. 992 \\
\hline & 0.270 & 79. 778 & 15.414 & 10. 335 & 2. 676 \\
\hline & 0.290 & 31.484 & -10.559 & -13.971 & -19.106 \\
\hline & 0.310 & 11.806 & -16.225 & -18.482 & -21.970 \\
\hline & 0.316 & 8. 652 & -16.267 & -18.259 & -21.373 \\
\hline & 0.320 & 6.988 & -16.072 & -17.905 & -20.796 \\
\hline & 0.340 & 2. 113 & -13.724 & -14.940 & -16.947 \\
\hline & 0.360 & 0.301 & -10.782 & -11.599 & -13.144 \\
\hline & 0.380 & -0.304 & -8.202 & -8.762 & -9.776 \\
\hline
\end{tabular}




\begin{tabular}{crrrrr}
\multicolumn{1}{c}{ Cont. table 1} & \multicolumn{1}{l}{} \\
\hline$\theta /\left(^{\circ}\right)$ & $R_{\mathrm{m}} / \mathrm{nm}$ & $\Delta E^{\mathrm{HF}} / \mathrm{cm}^{-1}$ & $\Delta E^{\mathrm{MP} 2} / \mathrm{cm}^{-1}$ & $\Delta E^{\mathrm{CCSD}} / \mathrm{cm}^{-1}$ & $\Delta E^{\mathrm{CCSD}(\mathrm{T})} / \mathrm{cm}^{-1}$ \\
\hline 180 & 0.250 & 186.002 & 82.123 & 76.308 & 64.544 \\
& 0.270 & 74.731 & 8.641 & 4.485 & -3.271 \\
& 0.290 & 29.090 & -13.821 & -16.667 & -21.836 \\
& 0.310 & 10.688 & -17.766 & -19.678 & -23.170 \\
& 0.316 & 7.763 & -17.495 & -19.189 & -22.304 \\
& 0.320 & 6.226 & -17.128 & -18.692 & -21.579 \\
& 0.340 & 1.758 & -14.219 & -15.270 & -17.268 \\
& 0.360 & 0.133 & -11.018 & -11.733 & -13.139 \\
& 0.380 & -0.387 & -8.319 & -8.813 & -9.820 \\
& & &
\end{tabular}

极小值在 $\theta=0^{\circ}$, 即 He-H-F 构型, $R_{\min }=0.316 \mathrm{~nm}$, 势能为 $-42.979 \mathrm{~cm}^{-1}$, 第二极小值在 $\theta=180^{\circ}$, 即 He-F-H 构型, $R_{\text {min }}=0.302 \mathrm{~nm}$, 势能为 -23.665 $\mathrm{cm}^{-1}$, 第一极小值的势阱深度约为第二极小值的势 阱深度的两倍, 这主要是由于 $\mathrm{F}$ 原子的半径比较小 的缘故, 后面将作进一步的解释。从第一极小值到 第二极小值存在一个鞍点, 其构型为 $\theta=90^{\circ}$, $R_{\min }=0.326 \mathrm{~nm}$, 势能为 $-15.141 \mathrm{~cm}^{-1}$. 相比之下, 势能面从 $\theta=0^{\circ}$ 到 $90^{\circ}$ 比从 $\theta=90^{\circ}$ 到 $180^{\circ}$ 较为平坦 . 从表 2 列出的不同角度、不同距离的相互作用能 数值的变化情况可以看出, MP2、CCSD 势能面上 也存在两个极小值 (在 2.2 部分给出了详细的计算 结果), 分别对应于 $\theta=0^{\circ}$ 和 $180^{\circ}$ 两个线型结构, 在 $\theta=90^{\circ}$ 处也有一个鞍点, 其势阱深度都要比 CCSD (T) 结果小; 而 $\mathrm{HF}$ 势能面上势能值大部分为正值. 表明三者用于此类势能面的研究都是不可靠的.

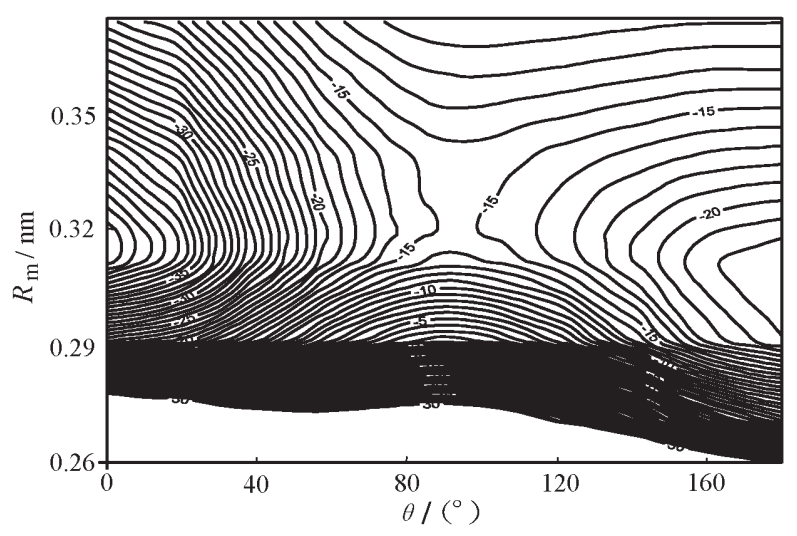

图 1 He-HF 体系势能面等势图

Fig. 1 Potential energy surface of the He-HF complex
虽然我们所采用的超分子方法没有将相互作 用能的各个部分 (交换、诱导和色散) 分离出来, 但 势能计算中 MP2、CCSD、CCSD (T) 和 HF 的结果相 差较大, 这从侧面反映出色散能是相互作用能的主 要部分. 相对于 HF 分子的电偶极矩和电四极矩, $\mathrm{HF}$ 分子的极化率和电离势要大得多. 众所周知, 极 化率和电离势是决定色散能的主要部分, 而电偶极 矩和电四极矩则对诱导能的大小起着关键的作用. 所以对此体系来讲，色散能是分子间相互作用能的 主要部分, 同时, 我们也看到势能面呈现出不是很 强的各向异性.

\section{2 基函数和理论方法的影响}

我们计算了各种理论方法、基函数下的相互作 用能的极小值和对应的 $R_{\mathrm{m}}$ 值, 见表 3. 基函数全部 采用 Dunning 基. 同时, 通过拟合公式 $A(x)=$ $A(\propto)+\alpha \mathrm{e}^{-\beta x}[A(x)$ 和 $A(\propto)$ 分别为复合物在

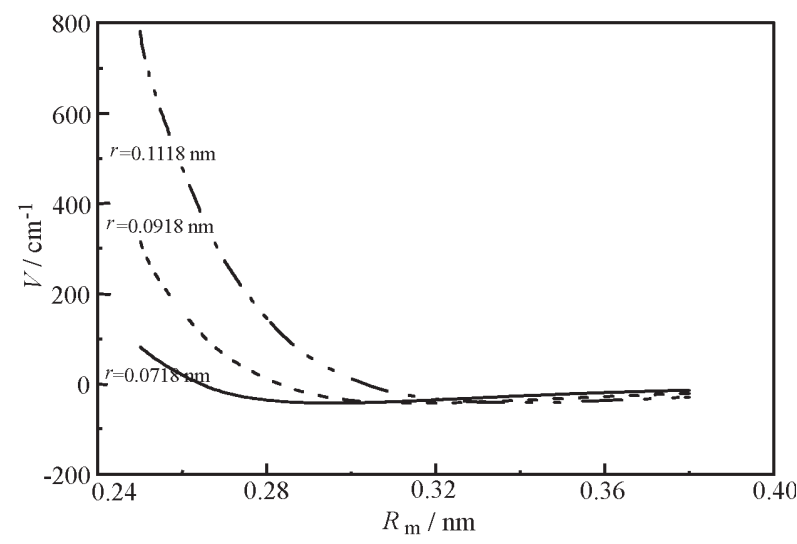

图 $2 \theta=0^{\circ}$ 时 $\mathbf{H}-\mathrm{F}$ 键长改变对势能的影响

Fig. 2 Effect of changing $\mathrm{H}-\mathrm{F}$ bond length on the interaction energy at $\theta=0^{\circ}$ 


\begin{tabular}{ccc}
$\begin{array}{r}\text { 表 } 2 \\
\text { Table } 2\end{array}$ & $\mathbf{0}^{\circ} 、 \mathbf{9 0 ^ { \circ }} 、 \mathbf{1 8 0}$ 的 $\boldsymbol{R}_{\min }$ 和 $\boldsymbol{V}_{\min }$ \\
\hline$\theta /(0)$ & $R_{\min } / \mathrm{nm}$ & $V_{\min } / \mathrm{cm}^{-1}$ \\
\hline 0 & 0.316 & -42.979 \\
90 & 0.326 & -15.141 \\
180 & 0.302 & -23.665 \\
\hline
\end{tabular}

aug-cc- $p \operatorname{VXZ}(X=D, T, Q, 5)$ 和极限基 (CBS)下的 相互作用能值, $\alpha, \beta$ 分别为拟合参数], 我们也给 出了复合物 He-H-F 和 He-F-H 在极限基 (CBS) 下 的相互作用能值 ${ }^{[16-17]}$. 从表 3 可以明显看出, 对复 合物的两种结构 $\mathrm{He}-\mathrm{H}-\mathrm{F}$ 和 He-F-H 来讲, 平衡距离 值 $R_{\mathrm{m}}$ 随基函数变化而变化的趋势是一致的，它们 都随基函数的增大而逐渐减小, 并且显示出了良好 的收玫行为, 特别是结构 He-H-F. 同时, 我们也看 到在 CCSD 理论级别下的 $R_{\mathrm{m}}$ 值比 MP2 级别下的 值要小一些, 但又大于 $\operatorname{CCSD}(\mathrm{T})$ 理论级别下的 $R_{\mathrm{m}}$ 值。这从另一个侧面说明对于此类复合物的从头 计算研究, CCSD 的结果要好于 MP2 的结果, 而最 好的结果为 $\operatorname{CCSD}(\mathrm{T})$ 值.

在 MP2、CCSD 和 $\operatorname{CCSD}(\mathrm{T})$ 理论级别下, 基函 数不同时的各相互作用能值其收敛行为与 $R_{\mathrm{m}}$ 非常 相似, 都是随基函数的加大而减小，在 MP2 和 CCSD 理论级别下的相互作用能值均较 $\operatorname{CCSD}(\mathrm{T})$ 值大, 又一次表明了 $\operatorname{CCSD}(\mathrm{T})$ 值为最好. 非常有趣
的是, 我们注意到对于不同的基函数, 无论是从相 互作用能值还是从 $R_{\mathrm{m}}$ 值上来看, CCSD 理论级别 下的值比 MP2 级别下的值都要小一些, 这说明 CCSD 理论级别下的计算效果要比 MP2 级别下的 好一些。这种情况在研究 $\mathrm{He}-\mathrm{HCl} 、 \mathrm{Ne}-\mathrm{HCl}$ 、 $\mathrm{He}-\mathrm{HBr}$ 和 $\mathrm{Ne}-\mathrm{HBr}$ 复合物时并没有出现。从表 3 可以看出, 对于此类非常弱的复合物计算来讲, 基 函数 aug-cc- $p V D T$ 和 aug-cc- $p V T Z$ 的质量不是很理 想, 而 aug-cc- $p V Q T$ 和 aug-cc- $p V 5 Z$ 下的值较为接 近.从分子间势的计算量和计算时间上来考虑, 基 函数 aug-cc- $p V Q Z$ 较为适合此类非常弱的分子间 相互作用形成的复合物分子间势的计算。所以, 本 文的计算除了用于比较之外都采用基函数 aug-cc-pVQZ.

\subsection{HF 键长 $r$ 对势能的影响}

为了考察 H-F 键长变化对势能的影响, 我们又 计算了 $\mathrm{H}-\mathrm{F}$ 键长分别为 $r=0.1118 \mathrm{~nm}$ 和 $r=$ $0.0718 \mathrm{~nm}, \theta=0^{\circ}$ 时的势能 (见表 4 及图 2).

从图 2 可以看出, $r$ 值的变化对势能带来明显 的影响, 但是分子间相互作用能的最小值 $V_{\text {min }}$ 却基 本保持不变; 当 $R<R_{\text {min }}$ 时,$r$ 的增加使势能增加; 当 $R>R_{\text {min }}$ 时, $r$ 的增加使势能降低. 其原因是在 $R<R_{\min }$ 时, $r$ 的增加使 $R$ 值相同时原子间距离更 近，在这一区域势能的主要部分是近程排斥能，所

表 3 复合物 He-HF 和 He-FH 的相互作用能和 $\boldsymbol{R}_{\mathrm{m}}$

Table 3 MP2, CCSD and CCSD(T) interaction energies and $R_{\mathrm{m}}$ of the He-HF and He-FH complexes

\begin{tabular}{cccc}
\hline & $\Delta E^{\mathrm{MP} 2} / \mathrm{cm}^{-1}$ & $\Delta E^{\mathrm{CCSD}} / \mathrm{cm}^{-1}$ & $\Delta E^{\mathrm{CCSD}(\mathrm{T})} / \mathrm{cm}^{-1}$ \\
\hline $\mathrm{He}-\mathrm{H}-\mathrm{F}$ & & & $-31.553(3.30)$ \\
aug-cc- $p V D Z$ & $-24.917(3.36)^{\mathrm{b}}$ & $-28.338(3.33)$ & $-39.215(3.19)$ \\
aug-cc- $p V D Z$ & $-29.141(3.26)$ & $-33.698(3.23)$ & $-42.979(3.16)$ \\
aug-cc- $p V D Z$ & $-32.148(3.23)$ & $-36.751(3.19)$ & $-44.828(3.152)$ \\
aug-cc- $p V D Z$ & $-34.289(3.221)$ & $-38.490(3.174)$ & $-46.614(3.149)$ \\
$\mathrm{CBS}$ & $-39.578(3.217)$ & $-40.791(3.163)$ & \\
$\mathrm{He}-\mathrm{F}-\mathrm{H}$ & $-11.180(3.25)$ & & $-14.094(3.20)$ \\
aug-cc- $p V D Z$ & $-16.025(3.12)$ & $-12.275(3.23)$ & $-21.169(3.05)$ \\
aug-cc- $p V D Z$ & $-17.770(3.09)$ & $-17.836(3.09)$ & $-23.665(3.02)$ \\
aug-cc- $p V D Z$ & $-18.398(3.083)$ & $-19.764(3.07)$ & $-24.546(3.014)$ \\
aug-cc- $p V D Z$ & $-18.752(3.081)$ & $-20.432(3.067)$ & $-25.026(3.012)$ \\
$\mathrm{CBS}$ & & $-20.787(3.067)$ & \\
\hline
\end{tabular}

a) The complete basis set.

b) The values of $R_{\mathrm{m}}$ are in parentheses $(\mathrm{nm})$. 
表 $4 \mathrm{H}-\mathbf{F}$ 键键长变化时的分子间作用能 $\left(\boldsymbol{V}_{\mathrm{m}}\right)^{\mathrm{a}}$

Table 4 Interaction energies $V_{\mathrm{m}}$ at different values of $r$ and $R$

\begin{tabular}{|c|c|c|c|c|c|c|}
\hline \multirow[b]{2}{*}{$r / \mathrm{nm}$} & \multicolumn{6}{|c|}{$V_{\mathrm{m}} / \mathrm{cm}^{-1}$} \\
\hline & $R / \mathrm{nm}$ & 0.27 & 0.32 & 0.34 & 0.36 & 0.38 \\
\hline 0.0718 & & -19.76 & -35.67 & -26.96 & -19.62 & -14.14 \\
\hline 0.0918 & & 63.2 & -42.69 & -36.53 & -28.19 & -20.91 \\
\hline 0.1118 & & 139.65 & -22.84 & -30.64 & -26.24 & -22.31 \\
\hline
\end{tabular}

以势能增大; 而当 $R>R_{\min }$ 时, 诱导能和色散能是 分子间相互作用能的主要部分, 对于这个体系而言, 色散能是主要部分.

\section{4 解析势能函数}

对于从头计算得到的离散点一般要将它们拟合 成一个解析函数形式才能方便地用于动力学和束缚 态计算. Hutson 研究组在这方面作了大量的、系统 的工作 ${ }^{[1,6-10]}$, 他曾提出了 H6 $(4,3,2) 、 \mathrm{H} 6 、 \mathrm{H} 4 、 \mathrm{M} 4$ 、 M5 等经验势, 取得了一定的效果. 对于弱相互作 用体系而言, 势能面一般都具有强度不等的各向异 性, 其解析函数形式一般采取对角度作球谐函数展 开的方法, 对径向部分, 采用把势能分为其各分量的 方法, 如近程排斥、诱导、色散等。对于稀有气体 卤化氢复合物, 本文采用以下带有一定数量可调参 数的解析函数形式:

$$
V_{\text {fit }}(R, \theta)=V_{\text {rep }}(R, \theta)+V_{\text {att }}(R, \theta)
$$

从上式可以看到, 势能简单的被分为排斥项和吸引 项两部分,其中排斥部分表达式为

$$
V_{\text {rep }}(R, \theta)=A \exp \left\{-\beta(\theta)\left[R-R_{0}(\theta)\right]\right\}
$$

势能中的吸引部分被描述为长程偶极 - 偶极相互作 用项，它的衰变与距离 $R^{-6}$ 相关。而偶极 - 四极相 互作用项的衰变与 $R^{-8}$ 相关.

$$
\begin{aligned}
V_{\text {att }}(R, \theta)= & -\frac{\left.f_{6} \beta(\theta) R\right) C_{6}(\theta)}{R^{6}} \\
& -\frac{\left.f_{8} \beta(\theta) R\right) C_{8}(\theta)}{R^{8}}
\end{aligned}
$$

阻尼函数 $f_{6}$ 和 $f_{8}$ 用于降低长程相互作用在 $R$ 较小 时对总的分子间相互作用能的影响, 它们采用如下 数学表达式 ${ }^{[19]}$ :

$$
f_{n}(x)=1-\exp (-x) \sum_{k=0}^{n} \frac{x^{k}}{k !}
$$

为了描述势能面中的各向异性,我们把方程(2)中的 可调参数 $\beta, R_{0}, C_{6}$ 以及 $C_{8}$ 作 Lengendre 多项式展
开, 并且考虑到对称性, 仅需对阶数奇偶性相同的 Lengendre 多项式进行展开.

$$
\begin{aligned}
\beta(\theta) & =\sum_{L=0}^{8} \beta_{L} P_{L}(\cos (\theta)) \\
R_{0}(\theta) & =\sum_{L=0}^{8} R_{0, L} P_{L}(\cos (\theta)) \\
C_{6}(\theta) & =\sum_{L=0}^{4} C_{6, L} P_{L}(\cos (\theta)) \\
C_{8}(\theta) & =\sum_{L=0}^{8} C_{8, L} P_{L}(\cos (\theta)
\end{aligned}
$$

这里 $P_{L}(\cos (\theta))$ 是 Lengendre 多项式的第 $L$ 个多 项式. 许多体系的研究表明, 上述表达式能够充分 精确地重现势能面 (PES) 的计算值.

于是，在整个的拟合过程中，我们一共使用了 17 个可调节参数 $\left(A 、 \beta_{0}, \beta_{2} 、 \beta_{4} 、 \beta_{6} 、 \beta_{8} 、 R_{0,0} 、 R_{0,2} 、\right.$ $R_{0,4} 、 R_{0,6} 、 R_{0,8} 、 C_{6,0} 、 C_{6,2} 、 C_{6,4} 、 C_{8,0} 、 C_{8,2} 、 C_{8,4}$ 、 $C_{8}$ ，6. 其中参数 $A$ 是固定的, 一般情况均采用一个 任意大值）. 这些参数为了最好地与势能面计算值 吻合被不断地调节. 基于这个目的, 我们定义如下 函数:

$F\left[\left\{\beta_{i}, R_{0, i}{ }^{\prime}, C_{6 j}, C_{8 j^{\prime}}\right\}\right]=$

$$
\sum_{l, l}\left|V_{\text {calc }}\left(R_{l}, \theta_{l^{\prime}}\right)-V_{\text {fit }}\left(R_{l}, \theta_{l^{\prime}}\right)\right|^{2}
$$

为了计算 $a b$ initio 势能面, 等式右边的求和要扩展 到整个一组 $\left\{R_{l}, \theta_{l}\right\}$.

一个非常有效的算法是用于极小化对应于一组 参数 $\left\{\beta_{i}, R_{0, i^{\prime}}, C_{6 j}, C_{8 j^{\prime}}\right\}$ 的函数 $F$. 这种方法可用 Monte Carlo 模拟退火程序及 Monte Carlo 动力学方 法来实现。通过上述方法, 我们拟合出的结果表明, 上述表达式能够充分精确地重现势能面 (PES) 的计 算值. 两者符合得非常好. 拟合出的数值和使用的 参数在此不再一一列举, 如有需要, 请与作者联系. 


\section{References}

1 Hutson M J. Annu. Rev. Phys. Chem., 1990, 123: 41

2 Gray G, Gubbins K E. Theory of Molecular Fluids, Vol

1. Fundamentals. Oxford: Clarendon Press, 1984: 293

Teachout R R, Pack R T. At. Data, 1971, 3: 195

Lovejoy C M, Nesbitt D J. J. Chem. Phys., 1990, 93: 5387

Lovejoy C M, Nesbitt D J. Chem. Phys. Lett., 1998, 147: 490

Hutson J M. J. Chem. Phys., 1988, 89: 4550

Hutson J M. J. Chem. Phys., 1992, 96: 6752

Hutson J M. J. Phys. Chem., 1992, 96: 4237

Hutson M J, Howard B J. Mol. Phys., 1981, 43: 493

Hutson M J, Howard B J. Mol. Phys., 1982, 45: 769

Novick S E. Bibliography of Rotational Spectra of Weakly Bound

Complexes, (2001). Electronic updates are available on the web

at http: //www. wesleyan. edu/chem/faculty/novick/vdw. html
12 Raghavachari K G W, Trucks G W, et al. Chem. Phys. Lett., 1989, 157: 479

13 Jr. Dunning T H. J. Chem. Phys., 1989, 90: 1007

14 Kendall R A, Jr. Dunning T H, Harrison R J. J. Chem. Phys., 1992, 96: 6796

15 Woon D E, Jr. Dunning T H. J. Chem. Phys., 1993, 98: 1358

16 Feller D. J. Chem. Phys., 1992, 96: 6104

17 Feyereisen M W, Feller D, Dixon D A. J. Phys. Chem., 1996, 100: 2993

18 Boys S F, Bernardi F. Mol. Phys., 1970, 19: 553

19 Frisch M J, et al. Gaussian 94. Gaussian, Inc., Pittsburgh PA: 1992

20 Tang K T, Toennies J P. J. Chem. Phys., 1984, 80: 3726

\title{
High Level ab Initio Study of Intermolecular Potential for the Rg-HX Complexs
}

Zhang Yu Shi Hong-Yun Wang Wei-Zhou

(Department of Chemistry, Guiyang University, Guiyang 550025)

\begin{abstract}
The potential energy surfaces of the ground state of the He-HF complex have been calculated at several levels of theory, including the single and double excitation coupled-cluster method with noniterative perturbation treatment of triple excitation $\operatorname{CCSD}(\mathrm{T})$. Calculations have been performed using the augmented correlation-consistent polarized quadruple zeta basis set (aug-cc- $p V Q Z$ ). Using the complete basis set (CBS), the global minimum with a well depth of approximately $46.614 \mathrm{~cm}^{-1}$ has been found for the linear He-H-F geometry $\left(\theta=0^{\circ}\right)$ with the distance $R_{\mathrm{m}}$ between the He atom and the center of mass of the HF molecule equal to $0.3149 \mathrm{~nm}$. In addition to the global minimum, there is a second minimum at $R=0.3012 \mathrm{~nm}$ and $\theta=180^{\circ}(\mathrm{a}$ well depth of $25.026 \mathrm{~cm}^{-1}$ ). The effects of the basis sets, $\mathrm{H}-\mathrm{F}$ bond length and theoretical methods on the intermolecular potential were discussed and a simple analytic form employing 17 adjustable parameters for fitting to the calculated PES was given.
\end{abstract}

Keywords: Ab initio, Intermolecular potential, PES, CBS

Received: June 18, 2001; Revised : August 10, 2001. Correspondent: Shi Hong-Yun(E-mail: hyshi@ tmail. gzu. edu. cn). * The Project Suppprted by the Educational and Scientific Commitee of Guizhou 